\title{
Study of novel high-density solar panels based on small space interconnection technology
}

\author{
Guoping Huang ${ }^{1}$, Hao Zhuang ${ }^{1,2, *}$, Honglie Shen ${ }^{2}$, Yashuai Jiang ${ }^{1}$, Guan $\mathrm{Sun}^{1}$, Xueliang \\ $\mathrm{Bai}^{1}$, and Jingnan $\mathrm{Li}^{1,3}$ \\ ${ }^{1}$ CECEP Solar Energy Technology (Zhenjiang) Co. Ltd, Zhenjiang, China \\ ${ }^{2}$ College of Materials Science \& Technology, Nanjing University of Aeronautics \& Astronautics, \\ Nanjing, China \\ ${ }^{3}$ CECEP Solar Energy Co. Ltd, Beijing, China
}

\begin{abstract}
Small space interconnection technology (SSIT) has been utilized in solar panels recently. The application of this technology can further increase the module efficiency. However, higher power decay after long-time operation may occur due to additional risk. Therefore, in this paper, damp heat (DH), thermal cycle (TC), UV irradiation and humidity freezing (HF) tests were utilized to study the weatherability performance of SSIT-based high-density solar panels fabricated with cells cut by two different techniques. The achieved data suggest that the novel high-density modules with small space between cells have good reliability in various weather conditions. The novel low-damage laser-induced cutting technique is beneficial for the improvement of module reliability.
\end{abstract}

\section{Introduction}

Technology of solar panels is progressing rapidly in the past several years[1]. The layout of a typical solar panel is glass/EVA/cell/EVA/backsheet. For a conventional panel, strings are formed by connecting cells with a space of around $2 \mathrm{~mm}$. Spurred by the demand for panels with higher efficiency, recently, high-density panel technology is under investigation. Benefited from this technology, space between cells could be reduced, which can decrease the inactive area in a string. However, the introduction of this small space interconnection technology in high-density panels may bring additional reliability risk.

Panel lifetime is directly correlated with the decay of panel output power. Various environmental conditions might lead to the power loss of solar panels, such as mechanical shock, ultraviolet irradiation, temperature change and high humidity [2-4]. Decay in power [5-8] is often triggered by these conditions that cause electrode corrosion, encapsulant discoloration, delamination between encapsulant layers and cell crackings. UV-induced decay is caused by the reduction in light transmittance of polymeric materials and power loss in solar cells. As different materials have different thermal expansion coefficients, big temperature variation can generate internal stress, which may consequently cause cell

\footnotetext{
* Corresponding author: zhuanghao@cecsec.cn
} 
crackings. Humidity degrades a panel by humid gas intrusion through the back encapsulant polymer, which consequently corrodes interconnecting ribbons and cell fingers.

Herein, we studied systematically the decay extent of these small-spaceinterconnection-technology (SSIT)-based solar panels in some typical environmental conditions. Damp heat (DH), thermal cycle (TC) test, ultraviolet (UV) irradiation and humidity freezing (HF) test were adopted to study the weatherability of these SSIT-based panels. After each test, the power of the panels under standard testing condition was measured. The achieved results demonstrate that these SSIT-based high-density panels have good weatherability in all these investigated environments. The novel low-damage laserinduced cutting technique is beneficial for the improvement of panel reliability. This work offers a reference for the massive application of SSIT-based high-density panels.

\section{Methods}

Sample panels were fabricated using standard production process with a typical glass/EVA/cell/EVA/backsheet structure. Damp heat, thermal cycling and UV sequential tests were conducted based on the IEC61215 standard methods utilizing Hongze HH84 equipment, HT484 equipment and Borui BR-PV-LID equipment, respectively. IEC61215 is an international standard generally adopted for the performance evaluation and qualification of solar panels. After each test, the power of sample panels was measured under STC $\left(25^{\circ} \mathrm{C}, 1000 \mathrm{~W} / \mathrm{m}^{2}, \mathrm{AM} 1.5\right)$.

\section{Results and discussion}

Half cell technology has been widely employed in solar panels currently. The mechanical damage of half cells during the cutting process is varied for different cutting techniques, which might influence the weathering performance of the SSIT-based high-density panels. If microcrackings are formed during the cutting process, the mechanical strength of the half cell will be much reduced. Thus, in this work, two different cutting techniques were adopted to evaluate their difference on panel reliability. Figure 1 shows the scheme of the small space interconnection technology. The space between cells in one string is $\sim 0.5 \mathrm{~mm}$.

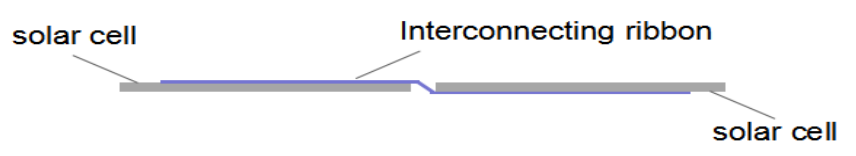

Fig. 1. Scheme of the small space interconnection technology.

Before weatherability performance evaluation, the initial property of the high-density solar panels based on small space interconnection technology was measured, see Table 1. Sample 11 to 13 were fabricated using conventional laser cutting technique. Sample 9, 10 and 16 were fabricated using a novel low-damage laser-induced cutting technique. The average power of sample 9,10 and 16 was $413.37 \mathrm{~W}$ and the average power of sample 11 to 13 was $415.58 \mathrm{~W}$. The unexpected lower power of sample 9, 10 and 16 might be caused by the long-trip transportation of the half cells, which were cut in the other factory. 
Table 1. Initial electrical property of high-density panels.

\begin{tabular}{cccccc}
\hline Panel & $\operatorname{Voc}(\mathrm{V})$ & $\operatorname{Vmp}(\mathrm{V})$ & $\operatorname{Isc}(\mathrm{A})$ & $\operatorname{Imp}(\mathrm{A})$ & $\operatorname{Pmp}(\mathrm{W})$ \\
\hline Sample 9 & 49.26 & 10.53 & 40.95 & 10.07 & 412.19 \\
Sample 10 & 49.34 & 10.51 & 41.16 & 10.07 & 414.49 \\
Sample 16 & 49.36 & 10.51 & 41.10 & 10.06 & 413.42 \\
Sample 11 & 49.44 & 10.52 & 41.25 & 10.07 & 415.45 \\
Sample 12 & 49.44 & 10.52 & 41.23 & 10.07 & 415.28 \\
Sample 13 & 49.47 & 10.52 & 41.29 & 10.08 & 416.02
\end{tabular}

To study the weatherability of these new high-density panels fabricated with cells cut by two different techniques in hot and humid climate, two panel samples were put in damp heat condition $\left(85^{\circ} \mathrm{C} / 85 \%\right.$ R.H.). Damp heat $(\mathrm{DH})$ test is often employed to evaluate the panel's weatherability to resist long-time intrusion of humid gas. Figure 2 shows the electrical performance under STC tested before and after DH. It can be noted that after $1000 \mathrm{~h}$ damp heat test, the power of sample 9 decayed from $412.19 \mathrm{~W}$ to $411.69 \mathrm{~W}$, sample 11 decayed from $415.45 \mathrm{~W}$ to $411.48 \mathrm{~W}$, implying good damp heat reliability for both panels. Panel fabricated with cells using the novel low-damage laser-induced cutting technique only degraded by $0.12 \%$, which is obviously lower than the one using the conventional laser cutting technique $(\sim 0.96 \%)$.

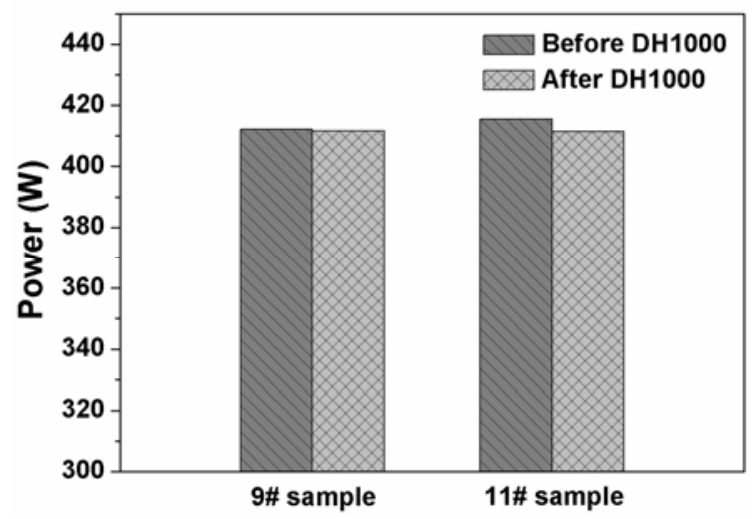

Fig. 2. Output power before and after 1000h DH test.

It is known that the characterization of dry heat environment is the big variation in temperature between noon and midnight. Thus, to inspect the weatherability of these new high-density panels fabricated with cells cut by two different techniques in dry heat environment, sample 10 was put in a simulated temperature change condition for 200 cycles from $-40^{\circ} \mathrm{C}$ to $85^{\circ} \mathrm{C}$. Figure 3 shows the panel output power before and after TC200 test. It could be noted after TC sample 10 with the novel low-damage laser-induced cutting technique decayed from $414.49 \mathrm{~W}$ to $413.23 \mathrm{~W}(-0.3 \%)$ and that of sample 6 decayed from $415.28 \mathrm{~W}$ to $413.59 \mathrm{~W}(-0.41 \%)$, demonstrating good dry heat withstand property of both panels and even better performance for the one using the novel low-damage laser-induced cutting technique. 


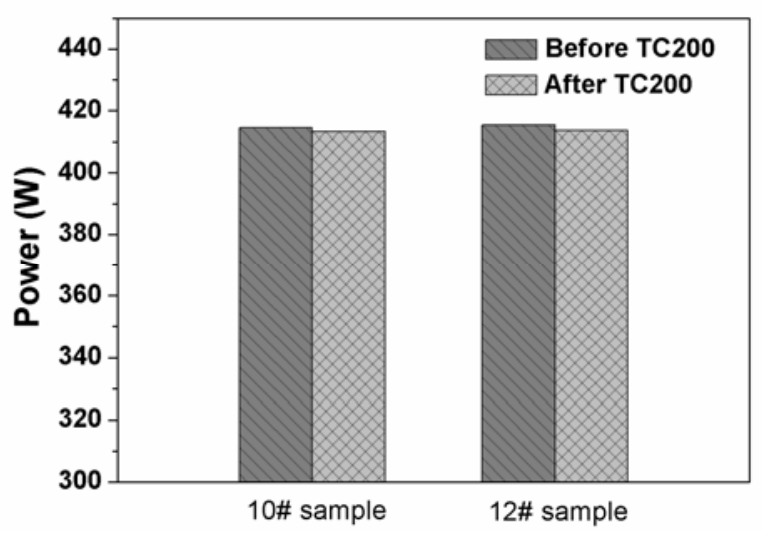

Fig. 3. Power of panels before and after thermal cycle test.

This new panel's weatherability was also studied in integrated condition. Sample panels were put in UV15-TC50-HF10 sequential test. Figure 4 shows measured power values before and after the sequential test. As can be seen, after $15 \mathrm{kwh}$ UV test, sample 13 decayed from $416.02 \mathrm{~W}$ to $410.02 \mathrm{~W}$, implying good UV resistant property. Afterwards, TC50 was carried out, sample 13 decayed from $410.02 \mathrm{~W}$ to $404.80 \mathrm{~W}$. After TC50 test, humidity freezing test was conducted on the module, after which the power was tested to be $396.99 \mathrm{~W}$. Therefore, after this sequential test, the overall power decay of sample 13 is $19.03 \mathrm{~W}$ (-4.58\%), exhibiting good weatherability in integrated condition. After UV test for $15 \mathrm{kwh}$, the power of sample 16 decayed $6.65 \mathrm{~W}$. Then TC50 test was conducted on the panel, the power of sample 16 increased from $406.77 \mathrm{~W}$ to $411.07 \mathrm{~W}$. Afterwards, humidity freezing test was conducted on the panel. The final power of the panel was $399.98 \mathrm{~W}$. Hence, after undergoing the sequential test, the overall power decay of sample 16 is $13.44 \mathrm{~W}(-3.25 \%)$, implying better weatherability of SSIT-based high-density modules fabricated with the low-damage laser-induced cell-cutting technique.

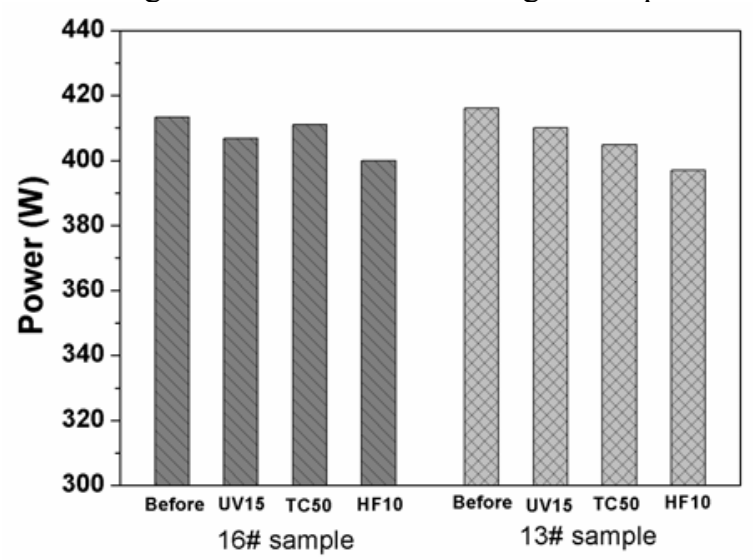

Fig. 4. Power of panels before and after sequential test.

\section{Conclusion}

Small space interconnection technology (SSIT) was applied in high-density panels. To demonstrate its weatherability, damp heat $(\mathrm{DH})$, thermal cycle (TC), UV irradiation and humidity freezing (HF) test were carried out. The power decay of panels with conventional 
laser cutting technique is $0.96 \%$ after DH1000 test, $0.41 \%$ after TC200 and $4.58 \%$ after UV15-TC50-HF10 sequential test. The decay of panels with novel low-damage laserinduced cutting technique is $0.12 \%$ after DH1000 test, $0.3 \%$ after TC200 and $3.25 \%$ after the sequential test. The achieved data verify that the fabricated new panels have excellent weatherability in damp heat, dry heat condition and after UV irradiation. Moreover, the novel low-damage laser-induced cutting technique is beneficial for the improvement of panel reliability.

\section{Acknowledgements}

Research program cecep-zdkj-2019-002 provided the financial support of this work.

\section{References}

1. Jibran Khan, Mudassar H. Arsalan, Solar power technologies for sustainable electricity generation - A review, Renewable and Sustainable Energy Reviews, 55, (2016)

2. Robert Witteck, Boris Veith - Wolf, Henning Schulte - Huxel, Arnaud Morlier, Malte R. Vogt, Marc Köntges, Rolf Brendel, UV - induced degradation of PERC solar modules with UV - transparent encapsulation materials, Progress in Photovoltaics, 25, (2017)

3. Alberto Pozza, Tony Sample, Crystalline silicon PV module degradation after 20 years of field exposure studied by electrical tests, electroluminescence, and LBIC, Progress in Photovoltaics, 24, (2016)

4. Yang Hu, Roger H. French, 5 - Degradation and Failure Mechanisms of PV Module Interconnects, Durability and Reliability of Polymers and Other Materials in Photovoltaic Modules, (2019)

5. Friederike Kersten, Peter Engelhart, Hans-Christoph Ploigt, Andrey Stekolnikov, Thomas Lindner, Florian Stenzel, Matthias Bartzsch, Andy Szpeth, Kai Petter, Johannes Heitmann, Jörg W.Müller, Degradation of multicrystalline silicon solar cells and modules after illumination at elevated temperature, 142, (2015)

6. Wei Luo, Yong Sheng Khoo, Peter Hacke, Volker Naumann, Dominik Lausch, Steven P. Harvey, Jai Prakash Singh, Jing Chai, Yan Wang, Armin G. Aberle and Seeram Ramakrishna, Potential-induced degradation in photovoltaic modules: a critical review, Energy and Environmental Science, 1, (2017)

7. Mahmoud Dhimish,Violeta Holmes, Bruce Mehrdadi, Mark Dales, The impact of cracks on photovoltaic power performance, Solar Energy Materials and Solar Cells, 2, (2017)

8. Taeko S, Corrosion mechanism analysis of the front-side metallization of a crystalline silicon PV module by a high-temperature and high-humidity test, Jpn. J. Appl. Phys. 59, 054001, (2020) 